BABELAO 3 (2014), p. 7-32

(C) ABELAO (Belgium)

\title{
Noms iraniens dans l'onomastique de la ville de Sardes
}

Par

\section{Fabrice Bouzid-Adler}

Doctorant à l'Université de Strasbourg

\begin{abstract}
A ncienne capitale des Mermnades, Sardes conserva à l'époque achéménide une grande importance et elle devint le cœur de la présence iranienne en Asie mineure occidentale au point que Bianor put écrire cette épigramme : "Sardes, qui pour le Roi fut la Perse en Asie mineure $»^{1}$. Edifiée sur les contreforts septentrionaux de la chaîne du Tmolos, la ville dominait la plaine de l'Hermos en contrebas et jouissait ainsi d'une situation très favorable au commerce. Son acropole construite sur une avancée rocheuse particulièrement escarpée constituait à la fois un poste de guet pour surveiller le pays et une défense réputée im-
\end{abstract}

1 Anthologie Palatine, IX, 423 (éd. P. Waltz, G. Soury, J. Irigoin et P. Laurens, CUF). Poète né en Bithynie, Bianor écrivit cette épigramme en évoquant le tremblement de terre qui ravagea Sardes en 16 de notre ère. 
prenable ${ }^{2}$. C'est sans doute en raison de ces atouts que les rois perses, reprenant à leur compte l'organisation mermnade, établirent à Sardes un satrape. Celui-ci, qui résidait vraisemblablement dans l'ancien palais de Crésus, tenait une cour brillante à l'image de la cour centrale et possédait des jardins situés entre le Mont Sipyle et la capitale lydienne ${ }^{3}$. Selon Xénophon, les satrapes devaient « obliger tous ceux qui recevraient une terre et une résidence officielle à fréquenter la cour $»^{4}$. En ce sens, on peut considérer les résidences satrapiques comme l'ossature de l'implantation iranienne dans les provinces conquises. Pour remplir sa tâche, le satrape était entouré d'une administration qui, pour une part au moins, devait être composée d'Iraniens. De plus, une garnison, commandée par un phrourarque, est attestée sur l'acropole de la ville durant tout l'empire. Le premier connu est Tabalos ${ }^{5}$ qui fut laissé par Cyrus le Grand à son départ de Sardes et qui résista sur l'acropole à une révolte lydienne menée par Paktyès. Au début du règne de Darius Ier, la garde du satrape Oroitès est estimée à un millier de Perses ${ }^{6}$. Plus tard, le phrourarque Orontas $^{7}$, nommé par Darius II, fut chargé par son successeur Artaxerxès Memnon de s'opposer aux prétentions de Cyrus le Jeune. Enfin, lors de l'invasion macédonienne, Mithrénès ${ }^{8}$ se rallia à Alexandre en entraînant la reddition sans combat de Sardes et de sa garnison. Il est remarquable que l'acropole ne fut jamais perdue pendant toute la durée de l'empire, ni lors de la révolte d'Ionie, ni après la victoire d'Agésilas sur le Pactole en 395 av. J.C. Son importance géostratégique était telle que les Perses œuvrèrent au mieux pour la contrôler. Ainsi, Strabon mentionne sur le Tmolos « une tourelle d'observation en marbre blanc, bâtie par les Perses, laquelle découvre toutes les plaines environnantes, et principalement la plaine du Caystre ${ }^{9}$.

\footnotetext{
${ }^{2}$ Les deux fois où l'acropole fut prise de vive force sont demeurées célèbres et elles doivent leur succès à des ruses : il s'agit de la prise de la ville par Cyrus en 547 av. J.-C. et celle par Antiochos III en 214 av. J.-C.

${ }^{3}$ DIODORE, Bibliothèque historique, XIV, 80, 1-2 : «Agésilas forma ses soldats en carré et poursuivit sa route au pied du mont Sipyle, guettant une occasion pour attaquer l'ennemi. Il traversa le pays jusqu'à Sardes et détruisit les vergers ainsi que le parc de Tissapherne, séjour où la végétation et les aménagements de toute sortes, réalisés avec art et à grand frais, invitaient à goûter aux charmes de la vie luxueuse et à jouir des plaisirs de la paix. » (éd. M. Bonnet et E. R. Bennet, CUF)).

${ }^{4}$ Xenophon, Cyropédie, VIII, 6, 10 (éd. E. Delebecque, CUF).

${ }^{5}$ Herodote, Histoires, I, 153.

${ }^{6}$ Herodote, Histoires, I, 127 ; Il n'est pas exclu que ce chiffre inclus à la fois la garnison et les Perses implantés autour de Sardes lesquels dépendaient $a$ priori du satrape en cas de guerre.

7 Xenophon, Anabase, I, 6, 6.

8 Arrien, Anabase, I, 17, 3-4 (traduction P. Savinel, 1984).

9 Strabon, Géographie, XIII, 4, 5 (traduction A. Tardieu, 1867).
} 


\section{Les "Perses de Sardes"}

La présence iranienne à Sardes ne se résume pas à ces quelques administrateurs et garnisaires. En effet, nous savons que dès le $\mathrm{VI}^{\text {ème }}$ siècle av. J.-C., des colons venus d'Iran se sont installés dans la ville. Les auteurs anciens les désignent sous le nom générique de "Perses de Sardes". Ils apparaissent, pour la première fois, dans notre documentation au début du règne de Darius Ier lorsque celui-ci leur ordonna, par l'intermédiaire de Bagaios, de tuer Oroitès coupable de trahison ${ }^{10}$. A l'époque de la révolte ionienne, Histiée fomenta des troubles parmi la population iranienne de Sardes et les encouragea à s'opposer au satrape Artaphernès.

[Histiée avait] envoyé des lettres à ceux des Perses qui se trouvaient à Sardes, comme à des hommes avec qui il avait eu, au sujet de la révolte, des conversations antérieures. Mais Hermippos, au lieu de donner ces lettres aux destinataires, les porta à Artaphernès et les lui remit entre les mains. Instruit de tout ce qui se passait, celui-ci ordonna à Hermippos de donner les messages d'Histiée à ceux à qui il devait les porter, mais de lui donner à lui les réponses que les Perses enverraient en retour à Histiée. Ces intrigues furent donc découvertes, et, à cette occasion, Artaphernès mit à mort un grand nombre de Perses. Tandis que des troubles se produisaient au pays de Sardes, (...). ${ }^{11}$

Les témoignages sur cette présence iranienne à Sardes viennent parfois de lieux inattendus. Ainsi, un papyrus araméen d'Egypte mentionne deux frères : Sprdy et Mtrnm soit peut-être *Spardiya (le Sardien) et ${ }^{*}$ Mithra-namab ${ }^{12}$. Tout porte à croire qu'il s'agit dès lors de deux Iraniens installés en Lydie qui séjournent en Egypte. La présence de ces colons dans la ville de Sardes est cependant difficile à constater archéologiquement. En effet, ce site a connu une forte iranisation culturelle et il est malaisé de faire la part entre implantation et acculturation. Plusieurs appliques en or, destinées à orner de riches vêtements témoignent ainsi de la diffusion de l'iconographie achéménide à Sardes. On notera, par exemple, deux sphinx barbus affrontés sous un disque ailé. Cette scène, encadrée par une frise à motif floral et surmontée de créneaux orientaux reprend des éléments courants dans l'art aulique susien. Une autre applique, montrant un lion androcéphale ailé a la particularité de montrer un visage imberbe : peut-être y a-t-il là une influence syrienne. Toutefois, l'empennage des ailes est un parfait décalque de celui des créatures représentées sur les murs des palais achéménides. Ces objets sont donc d'interprétation complexe. D'une part, leur lieu de fabrication est inconnu : sont-

${ }^{10}$ Herodote, Histoires, III, 128.

${ }^{11}$ Herodote, Histoires, VI, 4 (éd. Ph.-E. Legrand, CUF).

12 P. BRIANT, « Bulletin d'histoire achéménide I », Topoi Suppl. 1 (1997), p. 90. 
ils des productions locales, ce qui témoignerait d'orfèvres iraniens par leur origine ou leur formation artistique, ou d'objets venus du cœur de l'empire ? D'autre part, étaient-ils destinés à des colons issus de la diaspora ou à des aristocrates lydiens iranisés ?

La tombe pyramidale de Sardes pose des problèmes similaires d'interprétation. Elevée sur les bords du Pactole, elle présente des techniques de maçonneries lydiennes (comparables à celles employés dans les tumuli royaux de Bin Tepe) mais le bâtiment, lui, est sans équivalent en Lydie et il semble apparenté à la tombe de Cyrus à Pasargades. En dépit des débats pour reconstituer ce monument dont seules les six premières assises de calcaire sont conservées, il y a un consensus " pour dater ce monument de la fin du $\mathrm{VI}^{\mathrm{e}}$ siècle, et pour y voir une tombe, car il se situe au cœur d'une nécropole. ${ }^{13}$. G. M. A. Hanfmann a proposé d'y voir la tombe d'un noble iranien installé à Sardes ${ }^{14}$.

On le voit, la ville de Sardes a connu une forte iranisation sans doute causée par le côtoiement des communautés lydiennes et perses. Le présent article se propose d'étudier les noms iraniens portés par des Sardiens. Nous nous bornerons à considérer l'épigraphie qui est assez significative. En effet, l'onomastique de cette ville comprend, comme nous allons le voir, un nombre important de noms iraniens dont plusieurs sont contemporains de l'empire achéménide. De même que dans un précédent article portant sur la plaine hyrcanienne ${ }^{15}$, il s'agit de s'interroger sur les possibles apports de l'anthroponymie pour appréhender la diaspora impériale dans les provinces de l'empire perse. Outre les documents en langue grecque, nous traiterons des textes en langue lydienne, qui ont l'intérêt d'être pour la plupart de l'époque achéménide. Cette langue étant faiblement attestée et sa compréhension comportant encore maintes zones d'ombre, les traductions proposées doivent être considérées avec prudence ${ }^{16}$. Pour autant, nous verrons qu'elles ne manquent pas d'intérêt pour notre propos et qu'il eût été dommage de les exclure de cette étude.

13 G. LACAZE, « Lydie, au royaume de Crésus », Dossiers d'archéologie 328 (2008), p.38-51.

14 G.M. A. Hanfmann, Sardis, from Prehistoric to Roman Time, Cambridge (1983), p.103.

15 F. BOUZID-ADLER, «Anthroponymie iranienne dans la plaine hyrcanienne, un outil pour étudier la colonisation achéménide ? ", Bulletin de l'Académie Belge pour l'Etude des Langues Anciennes et Orientales 2 (2013), p. 73-75.

${ }^{16}$ Le problème se pose surtout pour les deux longues inscriptions de Mitridastaś fils de Mitratas. Les autres sont heureusement plus assurées. 


\section{Documents d'époque achéménide}

\section{A : Mitratas et son fils Mitridastaś}

Un corpus d'inscriptions lydiennes datant de l'époque achéménide nous fait connaitre une famille dont les deux membres connus portent des anthroponymes iraniens. Il s'agit d'un sceau au nom de Mitratas et de deux décrets émanant de son fils Mitridastaś, trouvées non loin du temple d'Artémis sur les rives du Pactole ${ }^{17}$.

\section{1 : Sceau de Mitratas}

Sceau conique à base orthogonale, de provenance inconnue, conservé au Department of Western Asiatic Antiquities du British Museum (Inv. $\mathrm{n}^{\circ}$ WA 115591). Il porte une inscription en lydien se déroulant autour d'un motif central. $2 \times 2 \mathrm{~cm}$. Datation : époque achéménide.

Editions : R. D. BARNETT, «A new inscribed lydian seal », Athenaeum 47 (1969), p.21-24 ; J. BOARDMAN, « Pyramidal stamps seals in the Persian Empire ", Iran 8 (1970), n²1 M. POETTO, " Un nuovo sigillo anatolico-persiano ", Kadmos 24 (1985), pp.83-85 ; E. R. M. Dusinberre, Aspects of Empire in Achaemenid Sardis, Cambridge, 2003, $\mathrm{n}^{\circ} 17$ (traduction anglaise seule).
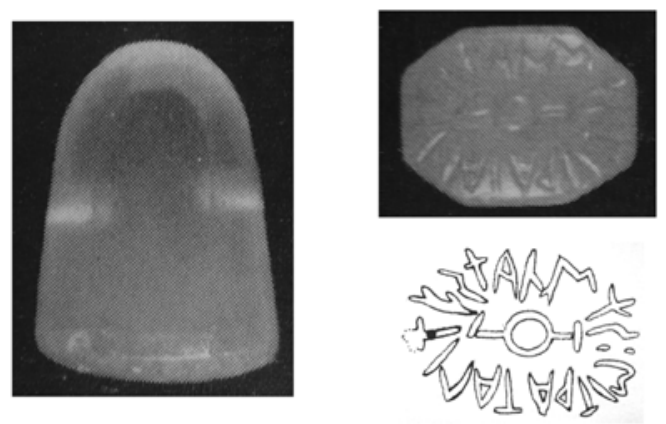

Sceau de Mitratas ${ }^{18}$

eś [s]admẽś : mitratali[s]

Ce sceau (est celui) de Mitratas.

La traduction pose relativement peu de difficulté. Le terme sadmẽś ${ }^{19}$, qui peut signifier "inscription" est aussi l'équivalent du

${ }^{17}$ Le rapprochement entre les inscriptions et le sceau est du à la rareté du nom Mitratas qui est inconnu par ailleurs en Lydie. Il s'agit donc simplement d'un argument de vraisemblance.

18 R. D. BARnETT, «A new inscribed lydian seal », Athenaeum 47 (1969), Pl. I, 4, 2 et 7 (reproduit avec l'aimable autorisation de la revue Athenaeum, Université de Pavie) 
grec $\sigma \tilde{\eta} \mu \alpha$ signifiant "signe" ou "marque". Dans ce contexte précis, le terme "sceau" semble tout indiqué. L’inscription encercle un symbole $(\mathbf{- O})$ dans lequel J. Boardman voyait un « personal blazon or identifying mark of the men named $»^{20}$. Peut-être s'agitil d'une ligature ${ }^{21}$ des lettres $1(\mathrm{l}), \mathbf{O}(\mathrm{o})$ et $\mathbf{T}(\mathrm{t})$ mais la signification générale reste obscure.

Concernant le nom même du propriétaire du sceau, il n'est pas possible de douter de son origine iranienne. Récemment, J. Tavernier proposait d'y voir la transcription de l'anthroponyme mède ${ }^{*}$ MiOräta ${ }^{22}$. Il s'agit clairement d'un nom théophore contenant le nom du dieu Mithra. Comme le soulignait R. Schmitt, la fin peut cependant être interprétée de diverses manières : hypocoristique formé par le suffixe -äta ou forme abrégée en *Mïra-t-a du nom *MiAra-tauxma ${ }^{23}$. Des parallèles existent en grec (M $\theta_{\varrho} \varrho \dot{\alpha} \tau \eta \varsigma$ notamment en Attique), en babylonien (Mi-it-ra-a-ta) ou en élamite (Mi-is-ra-da).

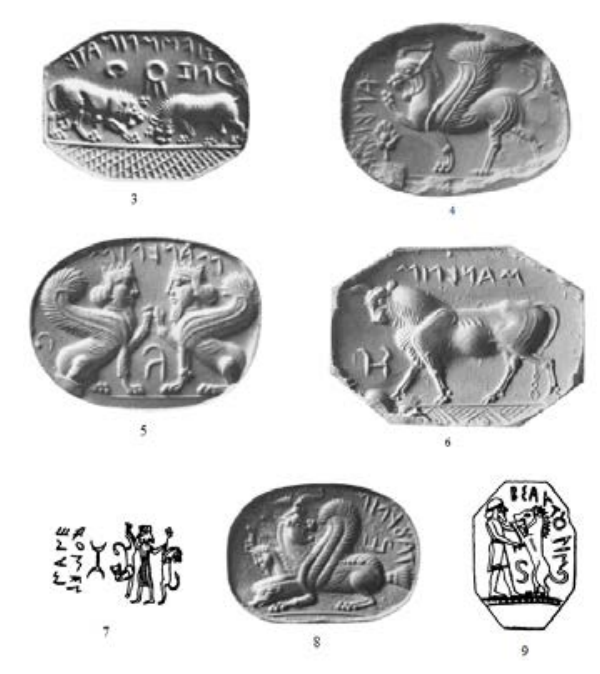

Iconographie achéménide sur des sceaux lydiens ${ }^{24}$

${ }^{19} \mathrm{La}$ lecture de la première lettre du mot sadmẽś pose problème : en effet, le signe normal devrait être $\boldsymbol{\mp}$ (s) au lieu de $\mathbf{+}$ (q). Il faut sans doute voir une erreur de l'artisan ayant gravé le sceau car le mot qadmẽś est inconnu par ailleurs.

${ }^{20} \mathrm{~J}$. BoARdman, «Pyramidal stamps seals in the Persian Empire », Iran 8 (1970), p. 21.

${ }^{21}$ R. D. BARNETT, «A new inscribed lydian seal », Athenaeum 47 (1969), p. 21 a établi un parallèle avec un autre sceau lydien conservé au Louvre (R. Gusmani, Lydisches Wörterbuch, mit grammatischer Skizze und Inschriftensammlung, Heidelberg (1964), n51). Celui-ci, marqué au nom de "Bakivaś fils de Samś", porte le signe $\boldsymbol{\sigma} \boldsymbol{\pi}$ qui est peut-être la ligature des lettres $\mathbf{q}(\mathrm{r}), \mathbf{\mathbf { x }}(\tau)$ et $\mathbf{J}$ (g).

22 J. TAVERNIER, Iranica in the Achaemenid period, Leuven (2007), p. 251.

${ }^{23}$ R. SCHMITT, Iranisches Personennamenbuch $V$, Wien (1982), p. IV/32.

${ }^{24}$ J. Boardman, "Pyramidal stamps seals in the Persian Empire », Iran 8 (1970), Fig. 2 et Pl. I (Creative Commons Attribution Non-Commercial NoDerivatives). 
Ce sceau est particulièrement intéressant si on le replace dans le corpus de sceaux pyramidaux à inscription lydienne établi par J. Boardman. Sur la dizaine d'exemplaires, plus de la moitié portent des représentations spécifiquement achéménides. On notera ainsi des animaux typiques du bestiaire susien (tels que des sphinx, des griffons, des lions et un taureau passant), un héros royal maitrisant des lions et un autre, en costume mède, tuant un autre fauve $e^{25}$. Pourtant, le seul sceau portant un nom iranien ne fait pas partie de ceux ayant cette iconographie iranisante. N'y a-t-il pour autant parmi les propriétaires des autres sceaux que des Lydiens ayant adopté des types issus de l'art aulique achéménide ? Rien n'est moins sûr. En effet, quatre sceaux ${ }^{26}$ portent une inscription selon laquelle ils appartiennent à un certain Manès. Longtemps considéré comme un anthroponyme, ce nom semble pouvoir être également un titre ${ }^{27}$. Dès lors, on peut suggérer que des Iraniens, dont les noms restent inconnus, aient été désignés sous le nom générique de Manès. Cette hypothèse demeure très fragile car elle suppose, d'une part, qu'il s'agisse bien d'un titre et, d'autre part, que des Iraniens l'aient porté.

\section{2 : Dédicace de Mitridastaś}

Bloc de calcaire découvert le 18 avril 1912 à 15 mètres environ au sud du temple d'Artémis, à un niveau un peu plus bas que celui-ci. Il est surmonté d'un fronton grossièrement taillé dans lequel sont gravés un croissant et une équerre de charpentier (ou une hache selon R. P. Scheil et B. Haussoulier). L'inscription devait figurer dans un rectangle de 41 sur $35 \mathrm{~cm}$. mais le lapicide semble avoir manqué d'espace car les lignes 1, 2, 8, 10 et 11 chevauchent cette limite. Les lettres sont dans l'ensemble bien conservées en dépit de quelques endroits où la pierre est abimée. le coin inférieur gauche est brisé. 79 × 56 × $29 \mathrm{~cm}$. Hauteur des lettres : entre 0,9 et $1,1 \mathrm{~cm}$. Datation : époque achéménide.

Editions : W. H. BUCKLER, Sardis VI, 2 : Lydian Inscriptions, Leiden (1924), $\mathrm{n}^{\circ} 23$; J. FrIEDRICH, Kleinasiatische Sprachdenkmäler, Berlin (1932), n²3; R. GUSMANI, Lydisches Wörterbuch, mit grammatischer Skizze und Inschriftensammlung, Heidelberg (1964), $\mathrm{n}^{\circ} 23$; E. R. M. DusINBERRE, Aspects of Empire in Achaemenid Sardis, Cambridge, 2003, $\mathrm{n}^{\circ} 13$ et pp. 119-120 (traduction anglaise seule).

Etudes et commentaires : L. ZGUSTA, «Iranian Names in Lydian Inscriptions », Charisteria Orientalia praecipue ad Persiam pertinentia (1956), pp. 397-398; R. D. Barnett, «A new inscribed lydian seal », Athenaeum 47 (1969), pp. 21-24.

1 eśś syrmaś Q $\lambda$ dãnl Artimu $\lambda \mathrm{k}$ dacuwerśt

2 wintad akit es $\lambda$ sirma $\lambda$ qys fẽns $\lambda$ ibid

3 niwiścv fakm $\lambda$ itin Q $\lambda$ dãnś tawśaś

$4 \quad$ Artimuk ibśimsis katsarlokid. brwãś

$5 \quad$ III II dãv ãn $\tau \tilde{t}$ Mitridastaś Mitratalis

${ }^{25}$ Respectivement les sceaux $n^{\circ} 5,4,8,3,6,7$ et 9 .

${ }^{26}$ Les sceaux $\mathrm{n}^{\circ} 4,5,6$ et 7 (pour ce dernier la lecture est incertaine).

${ }^{27}$ J. BOARDMAN, «Pyramidal stamps seals in the Persian Empire », Iran 8 (1970), p.21. 


\begin{tabular}{|c|c|}
\hline 6 & kaweś cito $\lambda s ́$ buk afariś q $\lambda$ dẽt amudãv \\
\hline 7 & akmav brafrsis brafrl $\lambda$ sawtarid fakud \\
\hline 8 & amu ow $v$ akit qed faśfẽnu akat $q \lambda$ fakantrow \\
\hline 9 & akm $\lambda$ is qis citollad bitad \\
\hline 10 & fakm $\lambda$ itin Q Q dãnk Artimuk katsarlokid \\
\hline 11 & bu $\lambda$ bill $\lambda$ arlyll $\lambda$ qyra $\lambda$ ẽtwerśkin \\
\hline 12 & sarokak esvav ciwav nikumẽk \\
\hline 13 & sawẽnt nik bis nik bilis arlyllis \\
\hline 14 & qysk amukit qyd faśfẽnu akat \\
\hline 15 & bawafu $\lambda$ sel $\lambda \mathrm{k}$ kantoru $\mathrm{a} \lambda \mathrm{a} \lambda$ nid \\
\hline 16 & $\mathrm{q} \lambda \mathrm{k}$ b $\lambda$ esak dẽt tafaaś alaś \\
\hline 17 & akit edkok ẽtqratad kotad amu \\
\hline 18 & Mitridastaś fẽtamvidv akś qis \\
\hline 19 & ẽmv etamv uv bawcvaśod buk in \\
\hline 20 & mẽtrid buk bidẽ $v$ qik int akm $\lambda$ \\
\hline 21 & qed ẽnud ẽtamś uv akad Artimuś \\
\hline 22 & biferśt \\
\hline
\end{tabular}

Ce temple est consacré à Q $\lambda$ dãns et Artémis, et si quelqu'un l'endommage par impiété, que le puissant Q $\lambda$ dãns et Artémis Ephésienne le détruisent. En l'année 5, tel est l'acte de donation que moi, le prêtre Mitridastaś fils de Mitratas, j’ai prescrit dans lequel j'accepte et je déclare : si je possède et que j'offre quoi que ce soit, si alors quelqu'un en abuse, que Q $\lambda$ dãns et Artémis, le détruisent lui et ses biens. Le scélérat ayant offensé ces dieux ne prospérera jamais, ni lui ni ses biens. Si je possède et que je confie quoi que ce soit à l'administration du sanctuaire et non à une autre personne, qu'un autre magistrat maintienne la propriété, alors cela sera accompli comme moi, Mitridastaś, je l'ai décrété. Et si quelqu'un mésestime mon décret ou lui nuit, alors, à ce décret que j’ai prescrit pour lui, qu'Artémis l'exécute.

En dépit des difficultés de traduction, la situation semble assez claire. En la cinquième année d'une ère inconnue, un prêtre nommé Mitridastaś consacre un temple à Q $\lambda$ dãns et Artémis d'Ephèse. Sans doute faut-il faut voir Apollon derrière le théonyme Q $\lambda d a ̃ n s$, ce qui expliquerait qu'il soit adoré dans le même temple qu'Artémis ${ }^{28}$. Le nom même de Mitridastaś a été interpréter de diverses manière ${ }^{29}$ : *MiOridasta ("Main de Mithra"), *Mïridāsta ("Accordé par Mithra") et *MiEridasta ("Donné par Mithra"). Dans tout les cas, il s'agit sans conteste dans nom mithriaque. Ce texte montre-t-il un noble Iranien ayant une dévotion à des dieux occidentaux ou s'agit-t-il d'une famille d'aristocrates lydiens qui auraient adopté des noms iraniens? L. Zgusta refusait l'origine iranienne de ce dédicant en arguant qu'une charge sacer-

${ }^{28}$ G.M. A. Hanfmann, Sardis, from Prehistoric to Roman Time, Cambridge (1983), p.94.

${ }^{29} \mathrm{Ni}$ R. SCHMITT, « Die theophoren Eigennamen mit altiranisch *MiOra- » in Etudes Mithriaques, Actes du 2e Congrès International, Téhéran, du $1^{\text {er }}$ au 8 septembre 1975 in Acta Iranica n ${ }^{\circ} 17$ (1978), p. 409 et Iranisches Personennamenbuch V, Wien (1982), p. IV/32 ; ni J. TAVERNIER, Iranica in the Achaemenid period, Leuven (2007), p. 485 ne tranchent cette question. 
dotale ne pouvait être dévolue qu'à des hommes issus de l'aristocratie lydienne ${ }^{30}$. Il voyait donc dans ce nom (et celui de son père Mitratas) une marque de l'iranisation des élites sardiennes adoptant des anthroponymes dans la langue de leurs maitres perses. Cette argumentation ne tient pas en raison des autres attestations dont nous disposons telles que le Mégabyze à Ephèse ou les Bagadatides à Amyzon, eux aussi prêtres d'un culte provincial. Il est en fait impossible de trancher. Le fait que Mitridastaś et son père portent des noms mithriaques n'est pas une preuve en soit de son origine iranienne mais, s'il était avéré que leur famille était issue de la diaspora impériale, cette inscription serait un nouvel indice suggérant que certains Perses de Sardes ont rapproché Mithra et Apollon ${ }^{31}$. Notons que, comme nous l'avons dit précédemment, l'anthroponyme Mitratas semble d'origine mède. Il n'est donc pas exclu, vu le contexte sacerdotal, que cette famille soit de la tribu des mages. Quelle que soit son origine, ce Mitridastaś fait partie de l'élite sardienne et il a à sa disposition des biens en suffisance. Le terrain sur lequel le temple est édifié semble lui appartenir et il l'offre aux divinités auxquelles il enjoint de punir quiconque ferait du tort au nouveau sanctuaire. Il évoque également des biens confiés aux administrateurs du temple. Il s'agit sans doute de dépendances permettant l'obtention de revenus réguliers. Peut-être avons-nous ici le décret fondant le sanctuaire qui abritera à l'époque hellénistique le temple d'Artémis encore visible de nos jours. En effet, c'est dans cette même zone proche du Pactole que furent trouvé, non seulement les décrets de Mitridastaś mais également un autel ${ }^{32}$ en grès rouge de 8,14 sur 8,82 mètres. Il a été daté de la période achéménide à la fois par les techniques de construction et par la présence de bols achéménides. La découverte de cet autel a été mis en relation avec un passage de Xénophon où Cyrus le Jeune évoque le serment prêté par Orontès sur l'autel d'Artémis ${ }^{33}$.

\section{3 : Les donations de Mitridastaś}

Bloc de marbre découvert le 11 avril 1914, à 50 mètres au sud du temple d'Artémis, en remploi dans une tombe d'époque byzantine. La pierre est assez endommagée surtout dans sa partie inférieure qui est brisée. $44 \times 23 \times 7 \mathrm{~cm}$. Hauteur des lettres : de 0,5 à $1,3 \mathrm{~cm}$. (lignes 1 à 12) et 0,4 à $1 \mathrm{~cm}$. (lignes13 à 23). Datation : époque achéménide.

${ }^{30}$ L. ZGustA, «Iranian Names in Lydian Inscriptions », Charisteria Orientalia praecipue ad Persam pertinentia (1956), p.397.

31 Dans la trilingue de Xanthos par exemple, le théonyme Apollon est traduit en araméen par le titre mithriaque Ḥ̌̌atrapati signifiant « Maître du pouvoir ». Cf. A. DUPONT-SOMMER, «La stèle trilingue récemment découverte au Létôon de Xanthos : le texte araméen ", Comptes-rendus des séances de l'Académie des Inscriptions et Belles-Lettres 118 (1974), p. 146.

32 E. R. M. Dusinberre, Aspects of Empire in Achaemenid Sardis, Cambridge, 2003, p. 60-64.

33 Xenophon, Anabase, I, 6, 7. 
Editions : W. H. BUCKLER, Sardis VI, 2 : Lydian Inscriptions, Leiden (1924), n²4 ; J. FrIEDRICH, Kleinasiatische Sprachdenkmäler, Berlin (1932), n²4; R. GUSMANI, Lydisches Wörterbuch, mit grammatischer Skizze und Inschriftensammlung, Heidelberg (1964), $\mathrm{n}^{\circ} 24$; E. R. M. DusINBERRE, Aspects of Empire in Achaemenid Sardis, Cambridge, 2003, p.119-120 et $n^{\circ} 14$ (traduction anglaise seule).

Etude et commentaires : R. D. BARNETT, «A new inscribed Lydian seal », Athenaeum 47 (1969), p.21-24.

\begin{tabular}{|c|c|}
\hline 1 & ãn ẽt Mitridastaś Mitra[talis] \\
\hline 2 & kaweś nak amu katosv faow \\
\hline 3 & akit nãqid serlis srmlis \\
\hline 4 & amu fadifil buktad amudãv \\
\hline 5 & fa[.]nal bukm $\lambda$ ad amu bitocv \\
\hline 6 & bid $v$ qedkm $\lambda$ a $\lambda$ ad fẽtamvid $v$ \\
\hline 7 & akm $\lambda$ is qis citollad bitaad \\
\hline 8 & bukm $\lambda$ is fakarsed qik bukm $\lambda \mathrm{a}[\mathrm{d}]$ \\
\hline 9 & fabuwerfta $\lambda$ bukm $\lambda$ it basvśak \\
\hline 10 & vãkid niwiśl $\lambda$ qel $\lambda \mathrm{k}$ buk \\
\hline 11 & mẽtlid buk bidẽv qik int \\
\hline 12 & fakm $\lambda$ it Artimuś ibśimsis \\
\hline 13 & katsarlokid $b \lambda$ bil $\lambda \mathrm{k}$ arlili \\
\hline 14 & qira $\lambda$ Artimu $\lambda$ kin ẽtwersn \\
\hline 15 & sarokak nikumẽk sawẽnt nik \\
\hline 16 & bis nik bil śfẽnis serlik srmli[s] \\
\hline 17 & ãn $\tau$ ẽt nak amu Mitridasta $\lambda s ́$ \\
\hline 18 & katos $v$ faow akit amu nãqid \\
\hline 19 & faśfẽnu nak aarav nak birak \\
\hline 20 & [n]ak dẽtn ẽm $v$ akat amu Mitridas[ta $\lambda]$ \\
\hline 21 & $[\mathrm{ka}] w e \lambda$ kantoru sawwaśtal akm[ $\lambda]$ \\
\hline 22 & [a入aś] nid qisk dẽt ifrlis Mitridasta $[\lambda]$ \\
\hline 23 & [env. 15 itad] Mitridastaś [- - -] \\
\hline
\end{tabular}

Tel est le décret prescrit et promulgué par moi, le prêtre Mitridastaś, fils de Mitra[tas] : si je confie quoi que ce soit à l'administration du temple ou que je [donne ?] ou met en gage quoi que ce soit d'autre destiné aux autres (?), si quelqu'un usurpe, divise, [...] ou [...] la donation par impiété ou lui cause du tort, que Artémis Ephèsienne le détruise lui et sa propriété. Le scélérat ayant offensé Artémis ne prospérera jamais, ni lui, ni ses biens. Tel est le décret prescrit et promulgué par moi, Mitridastaś, et l'administration du temple : si je possède quoi que ce soit, quelque cour ou quelque maison en ma propriété, et si je confie ces choses, m’appartenant à moi, Mitridastaś le prêtre, (...) un criminel (...) Mitridastaś (...) Mitridastaś (...).

Ce texte fait partie des inscriptions les plus longues en langue lydienne trouvées jusqu'à présent. Il semble qu'il comporte deux décrets (katosv) de contenu très proches concernant des donations de Mitridastaś. Le premier évoque la possibilité de confier des biens, notamment en gage, à l'administration du temple. Artémis Ephésienne doit protéger la donation contre les impies qui voudraient y porter atteinte. Le second décret, lacunaire, semble avoir 
la particularité d'avoir été promulgué à la fois par Mitridastaś et par l'administration du temple. Est-ce réellement une décision des deux partis ou est-ce une illusion due à une ambiguiité de traduction? Il s'agit en tout cas de confier des biens appartenant à Mitridastaś à la garde du sanctuaire. La seconde formule de malédiction est perdue mais il est probable qu'elle existait dans le texte originel.

\section{B : Inscription de Droaphernès}

Dédicace trouvée en 1973-1974 à Sardes sur les bords du Pactole. Gravée sur un bloc de marbre utilisé en remploi dans un bâtiment romain tardif. $45,5 \times 58,5 \mathrm{~cm}$ Hauteur des lettres d'environ $2 \mathrm{~cm}$. Datation : 427/426 ou 367-/366 av. J.-C.

Editions : L. ROBERT, « Une nouvelle inscription grecque de sardes : règlement de l'autorité perse relatif à un culte de Zeus ", Compte-rendu de l'Académie des Inscriptions et Belles-Lettres (1975) (= Opera Minora Selecta V, pp. 485-509) ; A. CHANIOTIS, Historie und Historikerin den griecbischen Inschriften. Epigraphische Beiträge zur griechischen Historiographie, Stuttgart (1988), D51 ; G. H. R. HORSLEY, New Documents Illustrating Early Cbristianity Vol. V Linguistic Essays, Sidney (1989), n ${ }^{\circ} 135$; P. BRIANT, « Droaphernès et la statue de Sardes ", Achaemenid History XI (1998) ; Bulletin Epigraphique, 1999 n 469; M. P. DE HOZ, Die lydischen Kulte im Lichte der griechischen Inschriften (Asia Minor Studien 36), Bonn (1999), 2.1; Supplementum Epigraphicum Graecum XL, 1071 ; XLI, 1009 ; XLIII, 692, 862 ; XLVI, 1531 ; XLVIII, 2188

Etudes et commentaires : Bulletin Epigraphique, 1976, nº624; C. H. Jr GREENEWALT, "The seventheeth campaign at Sardis (1974)", Annual of the American Society of Oriental Studies 43 (1978), p. 61-71; F. SOKOLOwSKI, «TA ЕМПҮРА : on the mysteries in the Lydian and Phrygian cults ", Zeischrift fuir Papyrologie und Epigraphik 34 (1979), p. 65-69 ; Supplementum Epigraphicum Graecum XXIX, 1205 et corrigenda XXX, 1897 ; M. BOYCE, A History of Zoroastrianism II : Under the Acbaemenids, Leiden (1982), p. 255-257 ; M. N. WEISKOPF, « Achaemenid systems of governing in Asia Minor », PhD, Berkeley I (1982), p. 98-107 ; P. FREI \& K. KOCH, Reichsidee und Reichsorganisation im Perserreich, Göttingen (1984), p. 19-21 (considère $B \alpha \varrho \alpha \delta \dot{\alpha} \tau \varepsilon \omega$ comme le génitif d'un anthroponyme) ; Supplementum Epigraphicum Graecum XXXV, 1253 ; P. BRIANT, « Les Iraniens d'Asie Mineure après la chute de l'Empire achéménide. À propos de l'inscription d'Amyzon ", Dialogues d'Histoire Ancienne 11 (1985), p. 180-181 (préfère voir ici une "survivance folklorique" plutôt qu'un culte immuable comme le supposait L. Robert) ; "Polythéisme et Empire unitaire (Remarques sur la politique religieuse des Achéménides)", Les Grandes Figures religieuses (1986) ; F. GSCHNITZER, « Eine persische Kultstiftung in Sardeis und die " Sippengötter » Vorderasiens » in K. Obenhuber, Im Bannkreis des Alten Orients (1986), p. 45-54 (rapproche "Zeus de Baradatès" d'un "Men de Pharnace" connu par Strabon, Géographie, XII, 3, 31) ; Supplementum Epigraphicum Graecum XXXVI, 1089 ; P. BRIANT, « Pouvoir central et polycentrisme culturel dans l'Empire achéménide (quelques réflexions et suggestions) », Achaemenid History I (1987), p. 17 et 20 ; M. N. WeISKOPF, «The so-called « Great Satraps' Revolt», 366-360 B.-C. », Historia Ein₹. 63 (1989), p. 91-93 ; M. L. CHAUMONT, « Un nouveau gouverneur de Sardes à l'époque achéménide d'après une inscription récemment découverte ", Syria 57/3 (1990), p. 579-608 (s’intéresse surtout à l'identité de Droaphernès) ; T. CORSTEN, « Herodot I.131 und die Einführung des Anahita-Kultes in Lydien ", Iranica Antiqua 26 (1991), p. 163-180 (cultes iraniens en Lydie) ; Bulletin Epigraphique 1993, nº184 ; M BOYCE \& F. GRENET, A History of Zoroastrianism III : Zoroastrianism under Macedonians and Romans rulers, Leiden (1992), p. 205 ; P. FREI, «Die persische Reichautorisation. Ein Überblick », Zeitschrift für Altorientalische und Biblische Rechtsgeschicbte 1 (1995), p. 1-35; P. BRIANT, Histoire de l'empire perse, Paris (1996), p. 696-697, 1025-1027 et 1035 ; 
P. FREI \& K. Koch, Reichsidee und Reichsorganisation im Perserreich 2, Göttingen (1996), p. 90-96 ; P. HERMAnN, « Mystenvereine in Sardeis », Chiron 26 (1996), p. 329-335 ; P. DEBORD, L'Asie mineure an IV siècle (412-433 av. J.-C.), Bordeaux (1999), p. 367-374 (compare les hypothèse de L. Robert et P. Briant).

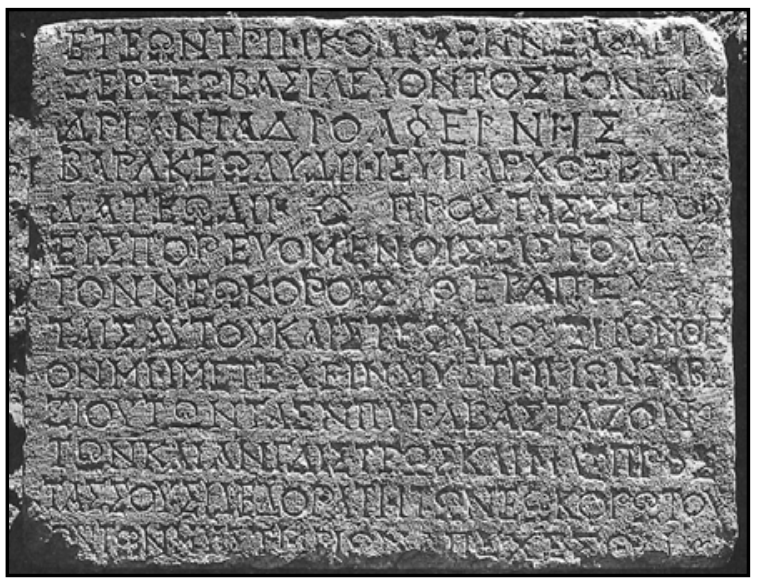

Inscription de Droaphernès ${ }^{34}$

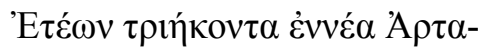

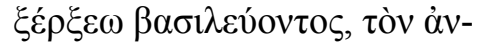
$\delta \rho i \alpha ́ v \tau \alpha \Delta \rho o \alpha \varphi \hat{\varepsilon} \rho v \eta \varsigma$

4 B $\mathrm{B} \rho(\alpha) \kappa \varepsilon \omega \Lambda v \delta i ́ \eta \varsigma$ vँ $\pi \alpha \rho \chi \rho \varsigma \mathrm{B} \alpha \rho \alpha-$

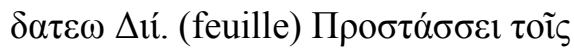

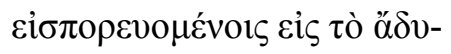

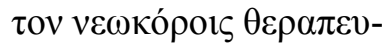

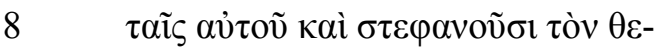

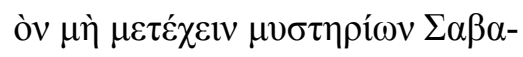

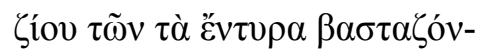

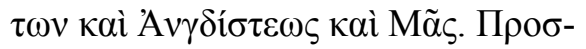

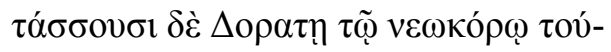

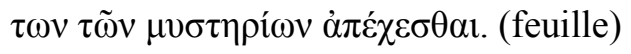

Année trente-neuf du règne d'Artaxerxès. Droaphernès, fils de Barakès, hyparque de Lydie (a consacré) la statue à Zeus de Baradatès. (feuille) Il interdit aux néocores desservants de ce dernier, qui ont le droit de pénétrer dans l'adyton et couronnent le dieu, de participer aux mystères de Sabazios de ceux qui apportent les victimes pour être brûlées, à ceux d'Angdistis et de Ma. Et il est fait interdiction au néocore Doratès de prendre part à ces mystères. (feuille) ${ }^{35}$

Cette inscription, regravée à l'époque romaine, comporte deux parties distinctes séparées par des feuilles : une dédicace et des interdictions cultuelles. La dédicace émane d'un certain Droa-

${ }^{34}$ L. ROBERT, «Une nouvelle inscription grecque de sardes : règlement de l'autorité perse relatif à un culte de Zeus ", Compte-rendu de l'Académie des Inscriptions et Belles-Lettres (1975), fig. 2 (reproduit avec l'aimable autorisation de l'Académie des Inscriptions et Belles-Lettres de Paris).

35 Cette traduction est empruntée à P. DEBORD, L'Asie mineure au IV siècle (412-433 av. J.-C.), Bordeaux (1999), p. 367. 
phernès, fils de Barakès. Son nom vient du vieux-perse *Druvafarnah qui signifie "Avec un éclat de gloire solide" et il porte le titre d'hyparque de Lydie en la trente-neuvième année d'Artaxerxès. Cette dernière indication permet d'établir une datation haute en 427/426 av. J.-C. (sous Artaxerxès Ier) et une datation basse en 367/366 av. J.-C. (sous Artaxerxès II). Selon l'hypothèse retenue, Droaphernès serait un hyparque subordonné soit à Pissouthnès, soit à Autophradatès. Bien que les commentateurs aient souvent opté pour la datation basse ${ }^{36}$, il faut reconnaitre que rien ne permet de trancher. Il s'agit, quoi qu'il en soit d'un Iranien de haut rang, pourvu d'une fonction officielle. Son père, Barakès, porte un nom pour le moins inhabituel qui viendrait du vieux-perse *Bara-ka, hypocoristique d'un nom en Barasignifiant "Celui qui porte, qui soutient". Le seul parallèle connu est fourni par un parchemin d'Avroman en langue parthe daté de 88-87 av. J.-C. ${ }^{37}$.

Dans la première partie de l'inscription, Droaphernès consacre une statue à Zeus dit « $\mathrm{B} \alpha \varrho \alpha \delta \dot{\alpha} \tau \varepsilon \omega$ ». Selon L. Robert, il s'agissait là d'un culte d'Ahura Mazda, le grand dieu iranien, hellénisé sous l'épithète de Zeus Législateur ${ }^{38}$. Il plaçait la dédicace de cette statue, qu'il supposait être celle de la divinité, dans la continuité d'une mesure du règne d'Artaxerxès II, connue par Bérose et transmise par Clément d'Alexandrie, qui consista à élever des statues cultuelles de la déesse Anahita dans les grandes villes de l'empire ${ }^{39}$. Depuis, il a été démontré que cette interprétation était erronée $^{40}$. Le terme $B \alpha \varrho \alpha \delta \dot{\alpha} \tau \varepsilon \omega$ n'est pas l'épithète de Zeus mais un génitif se rapportant à un anthroponyme iranien, B $\alpha \varrho \alpha \delta \dot{\alpha} \tau \eta \varsigma$ lequel est vraisemblablement l'ancêtre de Droaphernès. Baradatès est la transcription grecque de *Bara-däta signifiant "Qui donne la Loi" ${ }^{41}$. Ici, le terme "Loi" est à comprendre dans son sens religieux de loi divine. Des noms semblables, tels que B $\alpha \varrho \dot{\alpha} \delta \alpha \tau o \varsigma$, $\mathrm{B} \alpha \varrho \alpha \delta \dot{\alpha} \delta \eta \varsigma, \mathrm{B} \alpha \varrho \circ \delta \dot{\alpha} \delta \eta \varsigma$ et $\mathrm{B} \alpha \varrho \dot{\alpha} \delta \alpha \delta$ os, sont connus en Syrie aux $\mathrm{V}^{\text {ème }}$ et $\mathrm{VI}^{\text {ème }}$ siècles de notre ère ${ }^{42}$. Quoi qu'il en soit, puisqu'il s'agit d'un anthroponyme et non d'une épithète divine, le lien

${ }^{36}$ L. ROBERT, «Une nouvelle inscription grecque de sardes : règlement de l'autorité perse relatif à un culte de Zeus ", Compte-rendu de l'Académie des Inscriptions et Belles-Lettres (1975), M. L. CHAUMONT, « Un nouveau gouverneur de Sardes à l'époque achéménide d'après une inscription récemment découverte », Syria 57/3 (1990).

${ }^{37}$ M. MAYrhofer, « Zu den Parther-Namen der griechischen AvromanDokumente », Mémorial Jean de Menasce (1974), p. 209.

${ }^{38}$ L. ROBERT, «Une nouvelle inscription grecque de sardes : règlement de l'autorité perse relatif à un culte de Zeus ", Comptes-rendus de l'Académie des Inscriptions et Belles-Lettres (1975), p. 314.

${ }^{39}$ Clément D’Alexandrie, Proptétique, V, 65, 3.

${ }^{40}$ F. GSCHNITZER, « Eine persische Kultstiftung in Sardeis und die « Sippengötter »Vorderasiens » in K. Obenhuber, Im Bannkreis des Alten Orients (1986), p. 45-54 ; P. BRIANT, «Droaphernès et la statue de Sardes », Achaemenid History XI (1998).

${ }^{41}$ J. TAVERNIER, Iranica in the Achaemenid period, Leuven (2007), p. 132.

${ }^{42}$ L. ROBERT, "Une nouvelle inscription grecque de sardes : règlement de l'autorité perse relatif à un culte de Zeus ", Comptes-rendus de l'Académie des Inscriptions et Belles-Lettres (1975), p.314, n. 24. 
entre Zeus et Ahura Mazda n'a plus lieu d'être. Par conséquent, plutôt que l'introduction d'un culte iranien à Sardes, cette dédicace montre la piété d'un Perse de la diaspora impériale pour une divinité provinciale connue par ailleurs sous l'épithète de Zeus Polios.

Droaphernès consacre visiblement une statue humaine, le représentant probablement lui-même, à moins que ce ne soit son père ou son ancêtre supposé. Un parallèle existe dans la littérature ancienne car Diodore rapporte que les Macédoniens, sacrifiant à Athéna Ilias en Troade, virent " gisant à terre devant le temple, une statue d'Ariobarzanès, un ancien satrape de Phrygie ${ }^{43}$. Il est légitime de se demander si, en consacrant une statue à Zeus, Droaphernès n'avait pas d'autre motivation que la piété. En effet, ériger sa statue (ou celle d'un membre de sa famille) devant un sanctuaire indigène revenait, pour un aristocrate perse, à afficher sa bienveillance, et a fortiori sa domination, sur ledit sanctuaire. $\mathrm{Ph}$. Gauthier a souligné le fait que le dédicant a tenu «à préciser qu'il était un haut personnage de l'administration achéménide ${ }^{44}$ ce qui semble contraire à l'hypothèse d'un culte privé. Il y a donc tout lieu de penser que cette dévotion de Droaphernès s'inscrit dans un cadre politique surtout si l'on postule qu'il s'agit d'une stratégie familiale inaugurée par le dénommé Baradatès qui a peutêtre lui aussi exercé une charge au sein de l'administration satrapique. En tout cas, nous avons avec Droaphernès l'exemple, assez rare, d'un administrateur achéménide qui n'est pas envoyé du centre le l'empire mais choisi parmi les Perses de la diaspora.

La deuxième partie de l'inscription ${ }^{45}$ concerne des interdictions adressées au clergé assurant le culte de Zeus. Ceux-ci se voyaient interdits de participer aux mystères de Sabazios, d'Angdistis et de Ma. Toutefois, ces interdits ne sont peut-être pas à rattacher à la dédicace, ni à Droaphernès. En effet, ils semblent proches d'une autre inscription de Sardes ${ }^{46}$ se rapportant également aux néocores de Zeus. L'hypothèse de P. Briant ${ }^{47}$ est que, sous l'empire romain, les responsables du sanctuaire rassemblèrent, dans un souci « archivistique », divers documents de natures et d'époques variées et les firent regraver sur un mur en séparant chaque partie par une feuille. La pierre portant l'inscription de Droaphernès serait un des blocs de ce mur. Il n'est pas exclu que le texte original ait été en araméen. Toutefois, les exemples de Mitradastaś et de Droaphernès semblent montrer que les "Perses de Sardes" n'ont pas hésité à utiliser les langues provinciales (telles que le lydien et le grec) pour être compris par le plus grand nombre.

${ }^{43}$ Diodore, Bibliothèque historique, XVII, 17, 6.

44 Bulletin Epigraphique 1999, n 469.

${ }^{45}$ Chacune des parties étant séparé par une feuille.

46 W. H. BuCKLER \& D. M. ROBINSON, Sardis VII, 1 : Greek and Latin Inscriptions, Leiden (1932), n²2.

47 P. BRIANT, «Droaphernès et la statue de Sardes », Achaemenid History XI (1998). 


\section{C : Sardiens dans l'inscription des sacrilèges d'Ephèse}

Dédicace trouvée en 1973-1974 à Sardes sur les bords du Pactole. Gravée sur un bloc de marbre utilisé en remploi dans un bâtiment romain tardif. $45,5 \times 58,5 \mathrm{~cm}$ Hauteur des lettres d'environ $2 \mathrm{~cm}$. Datation : 427/426 ou 367-/366 av. J.-C.

Editions : F. EICHLER, «Die österreichischen Ausgrabungen in Ephesos im Jahre 1961 », Anzeiger der Osterreichischen Akademie der Wissenschaften 99 (1962), p. 50-52 ; D. KNIBBE, «Ein religiöser Frevel und seine Sühne : Ein Todesurteil hellenistischer Zeit aus Ephesos ", Jahreshefte des Österreichischen Archäologischen Institutes in Wien 46 (1964), p. 175-182 ; Bulletin Epigraphique, 1965, n³42 et $1981, \mathrm{n}^{\circ} 433$; H. WANKEL, Die Inschriften von Ephesos, Wien, 1979, $\mathrm{n}^{\circ} 2$; O. MASSON, "L'inscription d'Ephèse relative aux condamnées à mort de Sardes (I. Ephesos 2) », Revue des Etudes Grecques 100 (1987), p. 225-239 ; D. MCCABE R. N. ElliotT - A. Hilton - C. Redmond, Ephesos inscriptions. Texts and list, Princeton, $1991, \mathrm{n}^{\circ} 572$.

Etudes et commentaires : Bulletin Epigraphique, 1963, n²11 et $1965, \mathrm{n}^{\circ} 342$ ; F. SOKOLOWSKI, "A new testimony on the cult of Artemis of Ephesus ", Harvard Theological Review 58 (1965), p.427-431 ; Bulletin Epigraphique, 1966, n³69 ; L. ROBERT, «Sur des inscriptions d'Ephèse. Fêtes, Athlètes, Empereurs, Epigrammes », Revue de Philologie 41/1 (1967), p.32-36 (= Opera Minora Selecta V, 1989) ; Bulletin Epigraphique, 1967, n²498 ; H. WANKEL, « Die Bekanntmachung des Todesurteils in der ephesischen Inschrift Inv. 1631 », Zeischrift für Papyrologie und Epigraphik 24 (1977), p. 219-221 ; G. M. A. HANFMANN, "The Sacrilege Inscription : the ethnic, linguistic, social and religious situation at Sardis at the end of the Persian Era », Bulletin of Asia Institute 1 (1987), p.1-8 ; P. BRIANT, "Alexandre à Sardes ", Alexander the Great. Myth and reality, Annalecta Romana Instituti Danici Suppl. XXI (1993), p.1-15 ; Histoire de l'empire perse, Paris, 1996, p. 722-723, 1035 et 1068 ; F. BOUZID-ADLER, « Anthroponymie iranienne dans la plaine hyrcanienne, un outil pour étudier la colonisation achéménide ? ", Bulletin de l'Académie Belge pour l'Etude des Langues Anciennes et Orientales 2 (2013), p. 73-75.

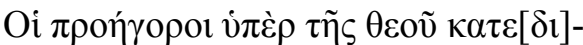

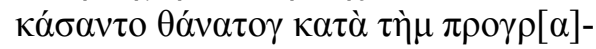

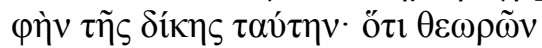

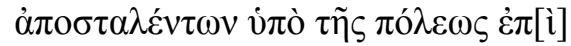

$5 \quad \chi 1 \tau \tilde{\omega} v \alpha \varsigma \tau \tilde{\imath} \iota$ A

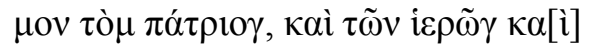

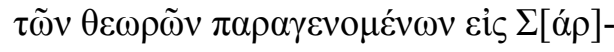

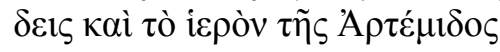

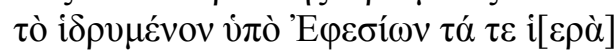

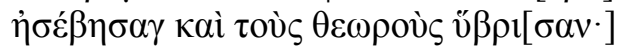

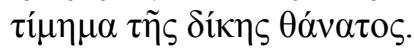

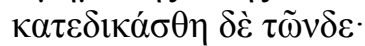

Suit la liste des condamnés dont:

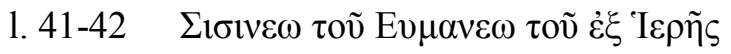

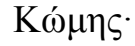

1. 46-47 Mi

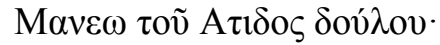

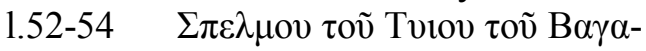

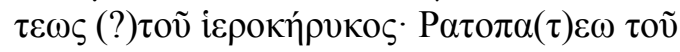
$\Pi \alpha \pi \varepsilon \omega$. 
Les défenseurs agissant pour la déesse, conformément à l'assignation au procès, ont réclamé des condamnations à mort. Attendu qu'après l'envoi [à Sardes] de théores par la ville, au sujet des tuniques pour Artémis, suivant l'usage ancestral, et qu'après l'arrivée des objets sacrés et des théores à Sardes et au sanctuaire d'Artémis - celui qui fut fondé par les Ephésiens - [des gens] ont commis des sacrilèges sur les objets sacrés et ont maltraité les théores, le verdict du procès est la mort. On a condamné les hommes que voici :

Suit la liste des condamnés dont :

1. 41-42 Sisinès fils d'Eumanès, celui de Hiéra Kômé.

1. 46-47 Mithradatès, de Tyios fils de Manès fils d'Atis, l'esclave

1.52-54 Spelmès fils de Tylos fils de Bagatès (?) - le héraut sacré ; Ratopatès fils de Papès ${ }^{48}$

Cette inscription, que nous avons déjà eu l'occasion d'évoquer à propos des noms iraniens de la plaine hyrcanienne est particulièrement utile pour étudier l'onomastique de Sardes. En effet, elle conserve les noms des quarante-cinq Sardiens s'étant rendus coupables de sacrilège envers la théorie. Cinq noms de cette liste sont d'origine iranienne. Tout d'abord est mentionné, aux lignes 41-42, un certain "Sisinès, fils d'Eumanès, celui de Hiéra Komè ». Les deux hommes résident dans un village de la plaine hyrcanienne. Toutefois, il convient de relever la précision « celui de Hiéra Komè » qui, à mon sens, indique qu'un autre Sisinès, fils d'Eumanès résidait probablement à Sardes. Sisinès est la transcription grecque du nom vieux-perse *Cicinah, hypocoristique en inah d'un anthroponyme construit sur le terme ${ }^{*}$ Cica signifiant "lignage" ou "brillant" ${ }^{49}$. Concernant le nom Eumanès, il vient du vieux-perse * $H u$ manah signifiant "Ayant de bonnes pensées" ${ }^{50}$. Parfois écrit *Vabu-Manah, ce nom est également connu par le babylonien $U$ hu-ma-na et le parthe Hwmny. Aux lignes 46-47 est mentionné Mithradatès, esclave de Tyios. Cette mention a l'intérêt de présenter un Iranien (ou le porteur d'un nom iranien) esclave d'un Lydien.

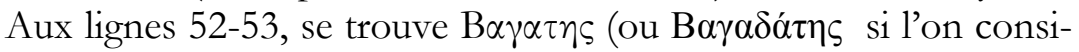
dère, avec $\mathrm{O}$. Masson, qu'il s'agit d'une erreur du lapicide $\left.{ }^{51}\right)$. Bagadatès est la transcription grecque de *Baga-dāta signifiant "Donné par Dieu"52. Cet anthroponyme iranien est également attesté en araméen $(B g d t)$, en babylonien (Ba-ga-'-da-a-ta) et en élamite (Ba-akda-ad-da). Si, à l'inverse, on refuse la correction de O. Masson, l'anthroponyme en question n'en est pas moins construit sur le terme vieux perse *Baga "Dieu". Enfin, la ligne 53 porte le nom

\footnotetext{
48 Cette traduction est empruntée à O. MASSON, «L'inscription d'Ephèse relative aux condamnées à mort de Sardes (I. Ephesos 2) ", Revue des Etudes grecques 100 (1987), pp. 227-228.

49 J. TAVERNIER, Iranica in the Achaemenid period, Leuven (2007), p. 551.

50 J. TAVERNIER, Iranica in the Achaemenid period, Leuven (2007), p. 199, 334 et 472 .

51 O. MASSON, «L'inscription d'Ephèse relative aux condamnées à mort de Sardes (I. Ephesos 2)», Revue des Etudes grecques 100 (1987), p. 227.

52 J. TAVERNIER, 2007, p. 132.
} 
РАТОПАПЕ $\Omega$ corrigé en PАТОПАТЕ $\Omega^{53}$. Il serait la transcription grecque de *Ratu-päta signifiant "Protégé par le Ratu". Selon E. Benveniste, "dans le mazdéisme avestique, le ratu est une autorité judiciaire, le patron du corps social, le garant de l'Ordre universel $»^{54}$. Notons que ce Ratopatès est fils de Papès. Ce nom est typiquement lydien ce qui pose des problèmes d'interprétation : cette famille est-elle iranienne, lydienne ou mixte ? Cette inscription est importante car, étant datée du dernier quart du IV ${ }^{\text {eme }}$ siècle av. J.-C., elle offre un nombre important de noms de Sardiens dans les années qui virent la chute de l'empire. Elle permet aussi de constater que les Iraniens de la diaspora n'étaient pas cantonnés dans l'élite de la ville.

\section{D : Epitaphe de (...) fils d'Artabãna}

Stèle trouvée en deux fragments en remploi dans un mur de la nécropole du Pactole. L'inscription, qui comptait originellement 12 lignes est en lydien. Un signe est visible en dessous du texte. $179 \times 39 \times 17,5 / 18,5 \mathrm{~cm}$. Hauteur des lettres : entre 0,75 et 2,75 $\mathrm{cm}$. Datation : époque achéménide (fin $\mathrm{V}^{\text {ème }}$ siècle av. J.-C. ?).

Editions : E. LITTMANN, VI, 1 : Lydian Inscriptions, Leiden (1916), $\mathrm{n}^{\circ} 9$; W. H. BUCKLER, Sardis VI, 2 : Lydian Inscriptions, Leiden (1924), $\mathrm{n}^{\circ} 8$; J. FRIEDRICH, Kleinasiatische Sprachdenkmäler, Berlin (1932), n8 ; R. GuSMANI, Lydisches Wörterbuch, mit grammatischer Skizze und Inschriftensammlung, Heidelberg (1964), $\mathrm{n}^{\circ} 8$.

$\begin{array}{ll}1 & \text { eśś wãnaś esk mrud e[s] } \\ 2 & \text { laqrisk kudkit es } \lambda \\ 3 & \text { [wã]na } \lambda \text { b } \lambda \text { tarwod aka[d] } \\ 4 & {[----]} \\ 5 & \text { Artabãnalid Katowal- } \\ 6 & \text { ik Atraśalid akit nãqis } \\ 7 & \text { fẽns } \lambda \text { ibid es } \lambda \text { wãna } \lambda \\ 8 & \text { buk es } \lambda \text { mru } \lambda \text { buk esv- } \\ 9 & \text { av laqrisav kudkit } \\ 10 & \text { es } \lambda \text { wãna } \lambda \text { b } \lambda \text { tarwod } \\ 11 & \text { fakav wiśśsis niwiścv } \\ 12 & \text { warbtokid }\end{array}$

Cette chambre funéraire, cette stèle, ce dromos [et] ce qui appartient à cette chambre funéraire [sont la possession] de (...) [fils] d'Artabãna et de Katovaś [fils] d'Atraśaś. Et si quiconque endommage cette chambre funéraire, cette stèle, ce dromos [et] ce qui appartient à cette chambre funéraire, qu'alors un [homme] pieux punisse son impiété.

\footnotetext{
53 O. Masson supposait que l'erreur était due à une confusion avec le nom suivant ПАПЕ (O. MASSON, "L'inscription d'Ephèse relative aux condamnées à mort de Sardes (I. Ephesos 2) », Revue des Etudes grecques 100 (1987), p.227 et 238).

${ }^{54}$ E. BENVENISTE, Titres et noms propres en iranien ancien, Paris (1966), p.105.
} 
Cette stèle, qui semble dater de la fin du $\mathrm{V}^{\text {ème }}$ siècle, appartenait à deux personnes dont l'un a un patronyme iranien (Artabãna). Ce nom, unique dans l'épigraphie anatolienne, est connu par les sources littéraires. En effet, plusieurs A $\rho \tau \alpha \dot{\beta} \beta \alpha v o \varsigma$ sont attestés dont le plus célèbre d'entre eux était le frère de Darius I ${ }^{\text {er55 }}$. Pendant longtemps, on admit l'étymologie *Rta-bānu "Ayant la splendeur d'Arta" ${ }^{56}$ mais, depuis une étude de R. Schmitt datant de 1980, on le rapproche plus volontiers de *Rta-pāna- "Sous la protection d'Arta" ${ }^{\prime 57}$ dont nous connaissons des transcriptions en vieil-indien (Atapāna), en parthe ('Rtpn) et en élamite (Ir-da-ba-na).

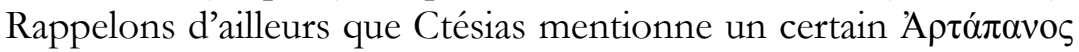

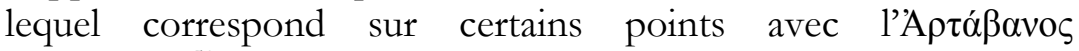
d'Hérodote ${ }^{58}$.

Le nom de du fils est, en revanche, manquant et il est impossible de le restituer. Tout juste pouvons-nous supposer, d'après la taille de la lacune, qu'il était assez long. Nous ignorons les liens existant entre lui et Katovaś, l'autre propriétaire de la tombe dont le nom est anatolien.

\section{E : Sceau d'Artimas}

Sceau-cylindre, de 2,2 cm., provenant de Lydie (l'origine exacte est incertaine) et portant une inscription araméenne. Il appartient à une collection privée. Datation : époque achéménide $\left(\mathrm{V}^{\text {ème }}-\mathrm{IV}^{\text {ème }}\right.$ siècle av. J.-C.).

Editions : A. D. H. BIVAR, «A "satrap" of Cyrus the Younger », Numismatic Chronicle (1961) ; P. BORDREuIL, "Sceaux inscrits des pays du Levant » in Dictionnaire de la Bible Supplément LXVI (1992), col. 86-212 (fig. 29) ; Corpus Inscriptionum Semiticarum, II, $\mathrm{n}^{\circ} 99$; E. LIPINSKI, Studies in Aramaic inscriptions and onomastics 1, Leuven (1975), p. 164-166

\section{'RTYM}

Artimas

L'inscription de ce sceau est trop lacunaire pour permettre un long commentaire. Le nom Artimas est peut-être iranien et c'est la raison pour laquelle il figure ici. Il serait un hypocoristique en -ima construit sur *Rta- ${ }^{59}$. Rappelons qu'Arta était, dans la pensée iranienne antique, la Vérité et la Justice divinisée. D’autres transcriptions de ce nom sont connues en babylonien (Ar-ti-im) et en éla-

55 Herodote, Histoires, IV, 83 (notamment) et Plutarque, De l'amour fraternel, 18.

56 Par exemple L. ZgusTA, "Iranian Names in Lydian Inscriptions », Charisteria Orientalia praecipue ad Persam pertinentia (1956), p.398.

57 R. SCHMITT, "Serta onomastica », Recherches de linguistique, Hommages à Maurice Leroy (1980), p. 199-200. C'est l'hypothèse reprise dans R. SCHMITT, Iranisches Personennamenbuch V, Wien (1982), p. IV/29 et J. TAVERNIER, Iranica in the Achaemenid period, Leuven (2007), p.299.

${ }^{58}$ CTÉsias, Persika, F13 \$24.

59 R. SCHMITT, Iranisches Personennamenbuch V, Wien (1982), p. IV/30 ; J. TAVERNIER, Iranica in the Achaemenid period, Leuven (2007), p. 306. 
mite (Ir-ti-ma). Certains ont voulu rapprocher ce sceau d'un passage de Xénophon mentionnant un certain Artimas, satrape de Lydie, à l'époque de la rébellion de Cyrus le Jeune ${ }^{60}$. Ce sceau serait donc possiblement le sien. Cette hypothèse est assez séduisante mais ne repose en fait que sur une analogie anthroponymique ce qui la rend fragile.

\section{Documents plus tardifs}

\section{A : Attestations autour du lac Gygès}

De par sa localisation à quelques kilomètres au nord de la capitale lydienne, la plaine entourant le lac Gygès peut être considérée ici, dans notre propos sur les attestations à Sardes. Cette plaine semble en effet avoir également vu l'implantation d'un peuplement iranien. La liste des conventus d'Ephèse ${ }^{61}$ nous fait ainsi connaitre, entre les cités de Daldis et de Julia Gordos, les 'Iov $\lambda \varepsilon i \tilde{s}$

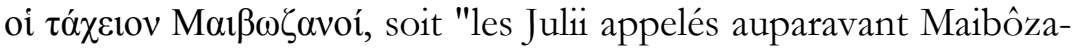
noi". Ch. Habicht y voyait un nom construit sur l'anthroponyme Maibouzanès et supposait que l'origine de cette localité était une colonie militaire perse ${ }^{62}$. L. Robert a proposé l'identification de cette ville avec l'agglomération moderne de Gölmarmara (qui tire son nom de la présence toute proche de carrières de marbre) ${ }^{63}$. En effet, A. Fontrier ${ }^{64}$ y avait découvert en 1886 une inscription monumentale indiquant l'existence d'un culte à Artémis Persique. Cette hypothèse est séduisante à condition que ladite inscription n'ait pas été déplacée à Gölmarmara depuis Hiérocésarée plus à l'ouest. Quoi qu'il en soit, il est incontestable que des Iraniens se sont installés dans les environs. Deux inscriptions, datés des deux premiers siècles avant notre ère, attestent de cette présence dans l'onomastique.

\section{1 : Liste de noms trowvée au sud du lac}

Bloc de marbre trouvé en 1922 au nord de Sardes sur la côte sud du lac Gygès, près du site supposé de Koloë. Brisé sur tous les côtés hormis sur le gauche où la marge de $2 \mathrm{~cm}$ est conservée. 49 × 39,5 x 22, 5 ; Hauteurs des lettres entre 7 et $13 \mathrm{~mm}$. Datation : fin $\mathrm{II}^{\mathrm{e}}$ - début $\mathrm{I}^{\text {er }}$ siècle av J.-C.

60 Xénophon, Anabase, VII, 8, 25.

${ }^{61} \mathrm{Ch}$. HABICHT, « New evidence on the province of Asia », Journal of Roman Studies, 65 (1975), p.64-91 et Pl. 1; G. P. Burton, 1975, p. 92-106 ; Bulletin Epigraphique, 1976, $\mathrm{n}^{\circ} 595 ; 1977, \mathrm{n}^{\circ} 450$; H. WANKEL, Die Inschriften von Ephesos Ia, Bonn (1979), n¹3 et Pl.14.

${ }^{62} \mathrm{Ch}$. HABICHT, « New evidence on the province of Asia », Journal of Roman Studies, 65 (1975), p.73-74.

${ }^{63}$ L. ROBERT, « Documents d'Asie Mineure », Bulletin de Correspondance Hellénique 106 (1982), p. 367-373.

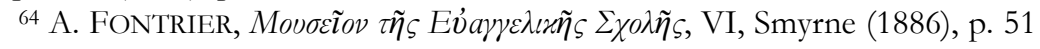
n532. 
Edition : W. H. BuckLer \& D. M. ROBINSON, Sardis VII, 1 : Greek and Latin Inscriptions, Leiden (1932), n5 (Pl. III) ; Supplementum Epigraphicum Graecum XXXII, n¹236.

Etudes et commentaires : L. ROBERT, Noms indigènes dans l'Asie mineure gréco-romaine I, Paris (1963), p. 82-83 (origine ethnique des différents noms) ; « Documents d'Asie Mineure », Bulletin de Correspondance Hellénique 106 (1982), p. 367 (rapproche cette liste de l'inscription des mystes d'Apollon Pleurénos).

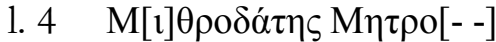 \\ Mithrodatès fils de Mètro(...)}

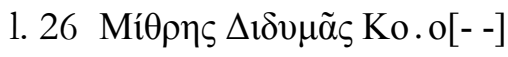

Mithrès Didymas fils de Ko(...)

\section{2 : Mystes d'Apollon Pleurénos}

Stèle à fronton découverte par G. M. A. Hanfmann dans un champ proche de Kemerdamlari, au nord-est du lac. Bien que brisée dans sa partie inférieure, il semble que les lacunes soient peu importantes. L'inscription, régulière dans ses quatorze premières lignes, est plus désordonnée par la suite. Elle est surmontée de deux rameaux de lauriers. 77 x 46 x 12cm. Datation : 26-25 av. J.-C.

Edition : L. ROBERT, « Documents d'Asie Mineure », Bulletin de Correspondance Hellénique 106 (1982), p. 361-367 (Supplementum Epigraphicum Graecum XXXII, n¹237)

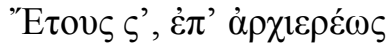

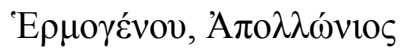

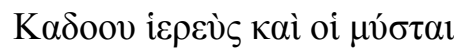

4 А

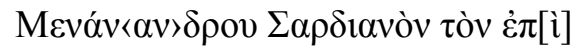

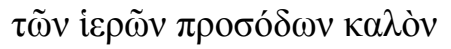

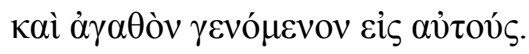

Suit la liste des myste dont :

1. $8-9$

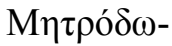

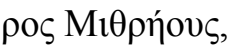

1. 14 Mí

En l'année 6, sous le grand-prêtre Hermogénès, le prêtre Apollonios fils de Kadoas et les mystes d'Apollon Pleurénos (ont consacré ceci) à Euxénos de Sardes, fils de Ménandros, qui, lors des processions sacrées, s'est conduit en homme de bien envers eux.

Suit la liste des myste dont :

1. 8-9 Métrodôros fils de Mithrès

1. 14 Mithrès fils de Métrodôros (...) 
Ces deux inscriptions nous semblent être liées comme l'avait proposé L. Robert et c'est pourquoi nous les traiterons conjointement. La seconde inscription ${ }^{65}$ émane d'une association cultuelle, les mystes d'Apollon Pleurénos, qui honore un certain Euxénos fils de Ménandros. Ce culte est sans doute à mettre en lien avec celui d'Artémis Koloénè dont le lac constituait le territoire sacré ${ }^{66}$. Faisant le lien avec la première inscription ${ }^{67}$, L. Robert proposait que ces deux stèles soient de même nature : dans les deux cas, il s'agirait de listes énumérant les noms de ceux qui avaient étés initiés aux mystères d'Apollon Pleurénos ${ }^{68}$.

Parmi les anthroponymes conservés par ces deux listes, on peut relever, dans la première inscription, datée de la fin du $\mathrm{II}^{\text {ème }}$ siècle av. J.-C., Mithrodatès et Mithrès Didymas tandis que la seconde inscription mentionne Métrodôros fils de Mithrès (lignes 89) et Mithrès fils de Métrodôros (ligne 14). Peut-être s'agit-il, comme le supposait L. Robert ${ }^{69}$, d'une même famille dont le grand-père et le petit-fils aurait porté le même nom, Métrodôros. Dès lors, l'anthroponyme mithriaque du père reste isolé et il ne prouve pas l'origine iranienne de cette famille. Tous les noms que nous avons relevés autour du lac Gygès sont construits à partir du théonyme Mithra et leur présence au sein de listes de mystes d'Apollon est troublante. De la même manière que pour les décrets de Mitradastas à Sardes, il est légitime de s'interroger sur un possible syncrétisme, aux yeux des Perses de la diaspora, entre les figures de ces deux divinités solaires liées à la fois à la lumière et à la vérité.

\section{B : Ménophantos fils de Mithrès}

Cette inscription fut découverte en 1914 par des paysans à Sardes au nord-ouest du temple d'Artémis, sur les bords du Pactole parmi les vestiges de murs présumés d'époque byzantine. Elle est datée de la dernière moitié $1 \mathrm{er} \mathrm{s}$ av. J.-C. 36 x 41 x 4cm. Hauteur des lettres $1 \mathrm{~cm}$. Datation : 50-51 av. J.-C.

Edition : W. H. BuCKLER \& D. M. RobINSON, Sardis VII, 1 : Greek and Latin Inscriptions, Leiden (1932), n¹32 ( fig. 119 ).

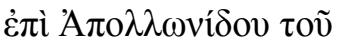

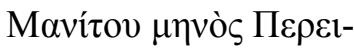 \\ $\tau \imath o v$ Mๆvó

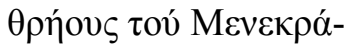 \\ $[\tau]$ ov $\dot{\varepsilon} \tau \tilde{\omega} \nu \vee \delta$.}

\footnotetext{
${ }^{65}$ Selon L. Robert, elle daterait de 26-25 av J.-C. ( Gème année de l'ère d'Actium).

${ }^{66}$ L. RoBert, « Documents d'Asie Mineure », Bulletin de Correspondance Hellénique 106 (1982), p.366.

${ }^{67}$ La numérotation adoptée ici est chronologique.

${ }^{68}$ L. ROBERT, «Documents d'Asie Mineure », Bulletin de Correspondance Hellénique 106 (1982), p.367.

${ }^{69}$ L. ROBERT, «Documents d'Asie Mineure », Bulletin de Correspondance Hellénique 106 (1982), p.362.
} 
Dans l'année où Apollonidès fils de Manitas était en fonction, au mois de Péreitios, (mourut) Ménophantos fils de Mithrès fils de Ménékratès, âgé de cinquante-quatre ans.

Cette inscription illustre la difficulté à utiliser l'onomastique comme marqueur ethnique. En effet, elle mentionne une famille dont le grand-père et le fils portent des noms grecs (Ménékratès et Ménophantos) et le père un nom iranien théophore (Mithrès). Devons-nous considérer qu'il s'agit d'une famille iranienne dans laquelle les noms grecs sont devenus nombreux en raison de l'hellénisation de Sardes ou d'une famille grecque où ce nom "Mithrès » aurait été donné sans connotation ethnique ? Il s'agit d'un nom iranien qui aurait eu peu de valeur démonstrative pour établir l'existence d'une présence perse s'il n'avait été trouvé sur un site où celle-ci est bien attestée par ailleurs.

\title{
C : Mithrès Kollubas dans des inscriptions de Syros
}

Le Rocher des Lettres est le promontoire occidental d'un petit port naturel au nord de $\operatorname{Syros}^{70}$ utilisé comme lieu de relâche aux époques antiques et médiévales. Une centaine d'inscriptions émanant des navigateurs y furent gravées au cours des siècles. Elles furent publiées par K. Stephanos qui les groupa en plusieurs corpus en fonction de leur localisation sur le promontoire. Le corpus $\Gamma$ ' comporte une dizaine d'inscriptions dont deux mentionnent le nom théophore Mithrès.

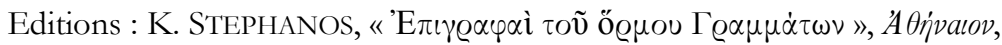
IV (1875), p. 3-32 (Inscriptiones Graecae XII, 5, 712, Г', 1-2); Bulletin Epigraphique 1956, n³ ; 1958, n45 ; L. RoBert, Documents de l'Asie Mineure Méridionale. Inscriptions, Monnaies et Géographie, Paris (1966), p.25 ; G. PFOHL, Griechische Inschriften als Zeugnisse des privatens und öffentlichen Lebens, München (1980), n¹44

Etude et commentaires : L. ROBERT, Hellenica VI, Paris (1948), p.11 et 1963, p.291 (traite du nom Kollubas)

1 : Inscription $\Gamma^{\prime} 1$

$$
\begin{aligned}
& \text { Mi } \theta \rho \tilde{\eta} \varsigma
\end{aligned}
$$

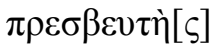

$$
\begin{aligned}
& \Sigma[\alpha \rho] \delta ı \alpha \text { ó } \varsigma
\end{aligned}
$$

Mithrès ambassadeur sardien.

\section{2 : Inscription $\Gamma^{\prime} 2$}

\author{
$\operatorname{Mi} \theta \rho \tilde{\eta} \varsigma \operatorname{Ko} \lambda \lambda v \beta \tilde{\alpha}[\varsigma$ ?]

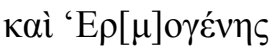 \\ $[\kappa] \alpha i ̀ ~ ' A \rho[\tau] \varepsilon \mu i ́ \delta[\omega] \rho \circ \varsigma$
}

70 Cette île se trouve dans les Cyclades, au milieu de la mer Egée. 
Mithrès Kollubas, Hermogénès et Artémidôros

En admettant que ces deux courtes inscriptions se réfèrent bien au même individu, nous pouvons apprendre ou supposer quel est le statut de ce Mithrès Kollubas. Son surnom peut, comme l'a montré L. Robert ${ }^{71}$, être mis en relation avec d'autres

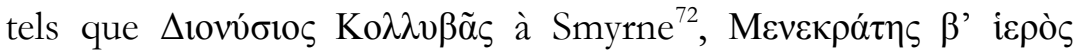
Ko $\lambda \lambda v \beta \tilde{\alpha} \varsigma$ à Magnésie du Sipyle ${ }^{73}$. Il se rapporterait «soit à la

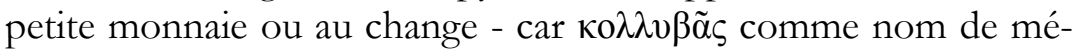

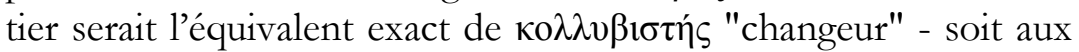
friandises $\gg{ }^{74}$. Dans le cas présent, il faut sans doute considérer Mithrès comme un riche sardien qui exercerait un métier lié au change et au commerce égéen (ce qui expliquerait la présence de son nom sur ce rocher). L'inscription $\Gamma^{\prime} 1$, nous apprend qu'il a été ambassadeur de Sardes dans un contexte qui demeure indéterminé. Etait-il d'origine iranienne comme son nom semble l'indiquer? Si tel est le cas, ces inscriptions montrent qu'après la chute de l'empire achéménide, des Iraniens de Sardes ont continué à avoir de l'importance à la fois sur le plan économique et sur le plan politique de la cité.

\section{$\mathrm{D}$ : Timbres amphoriques au nom de Mithrès}

Dès le début du $\mathrm{XX}^{\mathrm{ème}}$ siècle, les fouilles menées à Sardes ont permis de mettre au jour plusieurs fragments de céramiques au nom d'un certain Mithrès. En 1983, G. M. A. Hanfmann en comptabilisait vingt-quatre parmi les trouvailles de l'expédition Harvard-Cornell ${ }^{75}$. Il s'agit de céramiques sigillées orientales A et $\mathrm{B}$ trouvées dans les niveaux correspondant au I ${ }^{\text {er }}$ siècle av. J.-C.

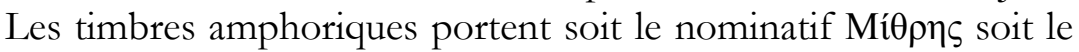

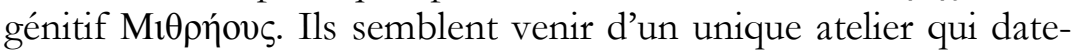
rait donc du début de la domination romaine. La production se compose de diverses céramiques d'usage courant telles que des pithos, des coupes et des bols. Aucun timbre amphorique au nom de Mithrès ne semblant avoir été découvert dans les autres sites, il est probable que cet atelier eut une audience très locale.

Quatre timbres amphoriques au nom de Mithrès sont présents dans le corpus de W. H. Buckler et D. M. Robinson publié $1932^{76}$. Il s'agit de ceux trouvés entre 1911 et 1914. Les relevés de G. H. Chase montrent une différence entre un timbre portant une légende au nominatif sur l'épaule d'un pithos (P242E) et trois autres

${ }^{71}$ L. ROBERT, Hellenica VI, Paris (1948), p.11 ; Noms indigènes dans l'Asie mineure gréco-romaine I, Paris (1963), p. 291.

72 B. V. HEAD, Catalogue of the greek coins of Ionia (BMC), London (1892), $\mathrm{n}^{\circ} 251,252$ et 254.

${ }^{73}$ L. ROBERT, Hellenica VI, Paris (1948), p. 11.

${ }^{74}$ L. RoBERT, Noms indigènes dans l'Asie mineure gréco-romaine I, Paris (1963), p.291.

75 G.M. A. Hanfmann, Sardis, from Prehistoric to Roman Time, Cambridge (1983), p.127.

76 W. H. BUCKLER \& D. M. ROBINSON, Sardis VII, 1 : Greek and Latin Inscriptions, Leiden (1932), $\mathrm{n}^{\circ} 224,7,10-12$. 
portant le nom au génitif. Ces derniers sont inscrits sur la face interne de petites céramiques : on compte ainsi une coupe (Ex 31a) ainsi que deux bols (Ex 17 et 28). Consulter les vingt autres exemplaires trouvés permettrait de confirmer (ou d'infirmer) cette distinction typologique entre timbres de grande et de petite céramique dans cet atelier. Quoi qu'il en soit, le Mithrès que nous fait connaitre ces timbres reste mystérieux. Peut-être s'agit-il d'un descendant de colons iraniens établis sous les Achéménides mais, en l'absence d'autres documents le concernant, nous ne pouvons que faire des hypothèses.

\section{E : Monétaires portant des noms iraniens}

Les trouvailles numismatiques ont permis de connaitre plusieurs magistrats monétaires sardiens portant des noms iraniens. Le premier est attesté à la fin de la période républicaine mais c'est pour l'époque impériale que nous avons le plus de documents.

\section{1 : Mithrès}

Une monnaie d'époque républicaine porte le nom du magistrat

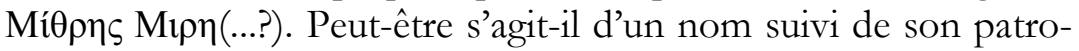
nyme soit Mithrès fils de Mirè(...). Ne connaissant aucun anthroponyme commençant ainsi, nous proposons de restituer un thêta entre le iota et le rhô. Le nom du monétaire serait donc peut-être Mí $\rho \eta \varsigma \mathrm{Mr}[\theta] \rho \eta ́[o u \varsigma]$ soit Mithrès fils de Mithrès. Bien que sujette à caution, cette hypothèse est assez plausible...

Publication : Th.-E. Mionnet, Description de Médailles Antiques, Grecques et Romaines: avec leur degré de rareté et leur estimation IV, Paris (1809), nº670.

- Au droit, Tête d'Artémis à droite, arc et carquois derrière le dos.

- Au revers, Athéna Pallas debout portant de la main droite une Nikè et ayant un bouclier posé à sa gauche ; à côté une lance :

CAP $\triangle \mathrm{IAN} \Omega \mathrm{N}, \mathrm{MI \Theta PHC} \mathrm{MI}(\Theta) \mathrm{PH}[\mathrm{OVC}]$

(Monnaie) des Sardiens, Mithrès fils de Mithrès (?).

\section{$2:$ Darius}

Plusieurs monnaies, émises pendant le règne d'Antonin le

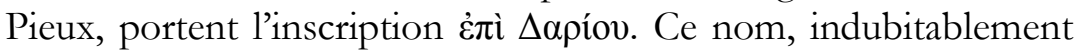
iranien, ne prouve pas une quelconque origine iranienne. En effet, les notables grecs ont parfois eu le goût de donner à leurs enfants des noms célèbres. Quoi qu'il en soit, nous connaissons cinq émissions monétaires au nom de Darius. Je ne reproduit ici que l'une d'entre elles à titre d'exemple datée entre 140 et 161 alors que Marc-Aurèle était César.

Publications : F. ImHOOF-BLumER, Lydische Stadtmünzen, Leipzig (1897) : Sardeis, $\mathrm{n}^{\circ} 14$, p.140 ; B. V. HEAD, Catalogue of the greek coins of Lydia (BMC), London (1901), Sardes, $n^{\circ} 141$, p.258 
- Au droit, buste de Marc Aurèle barbu à droite en tenue militaire M. AVPHAIOC KAICAP · Vח M(arc) Aurèle César.

- Au revers, couronne de laurier portant l'inscription : Darius.

CAP $\triangle I A N \Omega N$ EПI $\triangle \mathrm{APIOV}$ (Monnaie) des Sardiens, sous

\section{3: G(aius) Cl(audius) Mithros}

Un dernier magistrat monétaire portant un nom iranien, $\mathrm{G}$ (aius) $\mathrm{Cl}$ (audius) Mithros, est attesté sous Julia Domna, soit entre 193 et 217 de notre ère. Son gentilice indique qu'un de ses ancêtres obtint la citoyenneté sous les Claudii. Il est présenté par les inscriptions comme archonte de Sardes pour la première fois. Il faut sans doute comprendre qu'il s'agit d'une famille iranienne qui a su conserver une place éminente dans l'élite municipale sardienne. Nous ne reproduisons ici qu'une émission caractérisée par le type de Déméter (?) debout.

Publication : B. V. HEAD, Catalogue of the greek coins of Lydia (BMC), London (1901), Sardes, n¹47, p.259 (pl.XXVII,1).
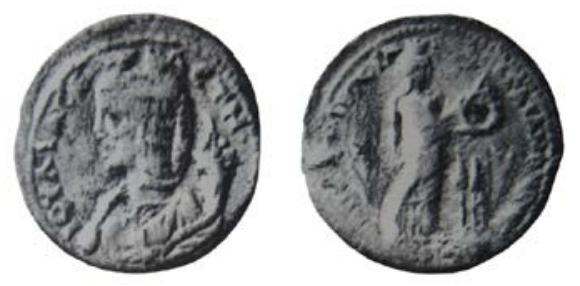

Monnaie de Julia Domna au type de Déméter debout ${ }^{77}$

- Au droit, buste de Julia Domna à gauche, portant comme Tychè un kalathos; corne d'abondance sur le côté gauche.

\section{IOYAIA $\cdot$ CEBACTH Julia Augusta}

- Au revers, Déméter (?) debout à droite, vêtue d'un long chiton et d'un péplos, tête voilée et surmontée d'un kalathos. La déesse tient devant elle de ses deux mains une couronne. Devant elle, deux épis de blé et derrière elle, un autre épi et un plant de pavot.

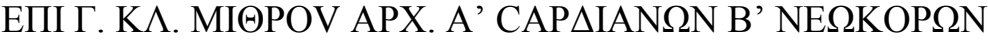

Sous $\mathrm{G}$ (aius) $\mathrm{Cl}$ (audius) Mithros, archonte pour la première fois,

(Monnaie) de Sardes, (cité) deux fois néocore.

77 B. V. HEAD, Catalogue of the greek coins of Lydia (BMC), London (1901), Sardes, Pl. XXVII,1. 


\section{Conclusions et perspectives}

L'onomastique de Sardes est sans conteste des plus intéressante pour quiconque s'intéresse à la présence iranienne en Asie mineure occidentale dans l'Antiquité. Par le nombre d'attestations, par leur répartition chronologique allant de l'époque achéménide à l'époque impériale et par les anthroponymes rares qu'il nous fait connaitre, ce corpus épigraphique illustre la réalité de l'implantation de populations iraniennes dans la capitale lydienne. Ces "Perses de Sardes" nous apparaissent dès lors sous un jour plus concret: nous les voyons en effet participer à des cultes locaux, utiliser des langues provinciales telles que le lydien et le grec et faire partie de familles apparemment mixtes. Certes, les incertitudes sont légion et la prudence est nécessaire pour appréhender ces documents. Après tout, certains de ces anthroponymes iraniens ont pu être portés par des indigènes intégrés dans le système satrapique. Cependant, une telle présence dans l'onomastique antique ne se comprend pleinement qu'en la mettant en lien avec l'existence, avérée dans la ville, de membres de la diaspora impériale.

Notons également que les documents contemporains de l'empire perse sont, de loin, les plus intéressants dans cette perspective à la fois parce qu'ils sont les plus pertinents pour étudier la colonisation achéménide et parce qu'ils fournissent des noms rares voire des hapax. Au contraire, les époques postérieures ne semblent connaitre qu'un nombre limité d'anthroponymes, souvent construits sur le théonyme Mithra. Il y a là sans nul doute la preuve d'une perte relative d'identité culturelle allant de pair avec l'hellénisation puis la romanisation. 